\title{
Pulsar Counterparts of Gamma-Ray Sources
}

\author{
P.A. Caraveo ${ }^{1}$ and G.F. Bignami ${ }^{2,1}$ \\ ${ }^{1}$ Istituto di Fisica Cosmica del CNR, Via Bassini, 15, 20133 Milano, Italy \\ ${ }^{2}$ Agenzia Spaziale Italiana, Via di Villa Patrizi 13, Roma, Italy
}

\begin{abstract}
The EGRET catalogue of unidentified X-ray sources has more objects along the galactic disk than at high galactic latitude, where identifications are comparatively easier. On the other hand, the Egret/GRO mission has already identified several known radio pulsars as gamma-ray sources as well as discovering Geminga's nature as a pulsar. If Geminga is not a unique case, as it is very likely not to be, than other galactic sources could, in fact, be radio quiet isolated neutron stars.

For these, the identification work is extremely difficult and should anyway start from high resolution X-ray/optical data.
\end{abstract}

\section{INTRODUCTION}

Isolated Neutron stars (INSs) are the only galactic objects surely identified as $\gamma$-ray emitters. Although not completely understood, as far as the emission mechanism is concerned, their phenomenology is fairly well known.

Combining multiwavelength observations spanning the entire electromagnetic spectrum, we have learned that diversity is the rule amongst $\gamma$-ray emitting INSs. With the notable exeption of the Crab, light curves are remarkably different in separate energy ranges (see e.g. Kanbach, 1997, for a review), hinting that alternate beaming geometries are possibly associated to different emission mechanisms. Moreover, the fraction of the rotational energy loss re-emitted in high-energy $\gamma$-rays ranges from a fraction of a percent, in the case of the Crab, to the quasi-totality for PSR1055-52 (see e.g. Goldoni et al, 1995). This does not translate directly into luminosity because the rotational energy loss of young pulsars is orders of magnitude larger than that of older objects, so that, in spite of lower efficiency, young pulsars are indeed the brightest $\gamma$-ray sources in our Galaxy.

With six successful identifications (counting also PSR 1915+32, which is not included in the $2^{\text {nd }}$ EGRET catalogue since it is detected only as a pulsating source) amongst the 45 low latitude sources seen by EGRET (Thompson et al, 1995, Thompson et al, 1996), it is natural to explore the possibility that at least a fraction of the remaining low latitude sources belong to the same class of compact objects. 
Indeed, many searches for pulsars inside COS-B and EGRET error boxes have been and are being carried out: all in all pulsars have received far more attention than any other galactic population thought to be a possible source of high-energy $\gamma$-rays. Why is the pulsar hypothesis so successful amongst $\gamma$-ray astronomers?

In a branch of astronomy hampered by poor angular resolution and low counting rates, a pulsar identification is by far the most unambiguous one. When the light curve obtained folding the $\gamma$-ray photons at a known pulsar period is statistically compelling, one should not worry about chance superposition nor about lengthy follow-up observations: the source is identified for sure.

This is the appeal of a pulsar identification and this is why pulsar searches have been performed on $\gamma$-ray error boxes as soon as COS-B discovered the UGOs (Unidentified Gamma Objects, see Bignami and Hermsen, 1983 for a review). The majority of the searches zeroed in on Geminga, but a fair number of the sources of the $2^{\text {nd }}$ COS-B catalogue were surveyed with no luck. However, one of the COS-B UGOs turned out to be a pulsar which was discovered in a routine survey for southern pulsars ten years after the end of the COS-B mission. It is the case of 2CG342-02, identified in 1992 with PSR 1706-44 (Thompson et al, 1992), a Vela-like pulsar at 2 kpc.

COS-B had two, with PSR 1706-44 three, radio pulsars amongst 22 low latitude sources. EGRET has five ( $\operatorname{six}$ if we count the tentative identification od PSR 0656+14 and seven if we consider also PSR 0540-69, seen by all CGRO instruments but EGRET) amongst 45 sources. In spite of the increased sensitivity of EGRET, the ratio between pulsar identification and total number of sources appears to be constant. Also the lack of results, experienced at the time of COS-B, appears to be unchanged. Dedicated radio searches (Nice and Sayer, 1997), aimed precisely at the search for radio pulsars inside the error boxes of 10 of the brightest EGRET sources, yielded null results, showing that the straightforward radio pulsar identification is not the only possible solution to the enigma of the unidentified high-energy $\gamma$-ray sources. This is further strengthned by the work of Nel et al. (1997) who investigated 350 known pulsars finding few positional coincidences but no significant timing signature for any of the pulsars in the survey.

\section{A DIFFERENT APPROACH}

Indeed, $\gamma$-ray astronomy does offer a remarkable example of an Isolated Neutron Star (INS) which behaves as a pulsar as far as X-and- $\gamma$ astronomy are concerned but has little, if at all, radio emission. As an established representative of the non-radioloud INSs (see Caraveo, Bignami and Trümper, 1996 for a review), Geminga offers a more elusive template behaviour: prominent in high energy $\gamma$-rays, uneventful in Xrays and downright faint in optical, with sporadic or no radio emission. Although the latitude distribution of unidentified EGRET sources shows that, in average, they are at least 10 times as distant as Geminga (Mukherjee et al, 1995), the multiwavelength behaviour of this source is hard to beat when one tries to link 
gamma-ray sources to compact objects in the absence of a radio signal. While one should always keep a totally open mind and be ready to find something new and different, Geminga is the template observers have in mind when planning observing strategies. Unfortunately, in spite of our knowledge of the behaviour of the real Geminga, the study of a Geminga-like source still represents a great challenge to observers since it defies well established techniques. To appreciate such a challenge let us briefly review the many steps that lead to the identification and the understanding of this object with an aim to find the signature to look for.

\section{THE MANY FIRSTS OF GEMINGA}

Briefly, the source was discovered in high energy $\gamma$-ray by the SAS- 2 satellite in 1972 (Fichtel et al, 1975), an X-ray counterpart, suggesting position and distance, has been proposed in 1983 (Bignami et al. 1983) and an optical one, refining the position, in 1987/88 (Bignami et al, 1987, Halpern and Tytler, 1988). However, the breakthrough came with the discovery of the $237 \mathrm{msec}$ periodicity in the ROSAT data (Halpern and Holt, 1992). Finding the same periodicity in the simultaneous high energy $\gamma$-ray data of the EGRET instrument (Bertch et al, 1992), as well as in the old archival COS-B (Bignami and Caraveo, 1992) and SAS-2 data (Mattox et al, 1992), yielded the value of the period derivative and thus of the object's energetics. The discovery of the proper motion of the proposed optical counterpart (Bignami et al, 1993) confirmed the optical identification and, thus, provided the absolute positioning of Geminga to within the systematic uncertainty of the Guide Star Catalogue, i.e. 1". Next came the measure of the source parallactic displacement, yielding a precise measure of its distance (Caraveo et al, 1996).

More HST observations, confirming and refining difficult measurements with ground-based instruments, have shown that a broad feature, centered at $\lambda=5998 \AA$ and with a width of $1,300 \AA$, is superimposed to the Rayleigh-Jeans continuum, as extrapolated from the soft X-rays (Bignami et al, 1996; Bignami, 1997). If interpreted as an ion-cyclotron emission, it implies, for $\mathrm{Z} / \mathrm{A}=1$, a $\mathrm{B}$ field of $3.2510^{11}$ Gauss as opposed to the value of $1.510^{12}$ obtained, theoretically, using the Period and Period derivative. This is the first time that the magnetic field of a neutron star is directly measured. Recently, the phenomenology of the source at high energies has been considerably enriched, owing to the very precise positioning of the optical counterpart. The possibility to link HST data to the Hipparcos reference frame, yielded the position of Geminga to an accuracy of 0.040 arcsec, a value unheard of for the optical position of a pulsar, or of an object this faint (Caraveo et al, 1997). This positional accuracy has allowed to phase together data collected over more than 20 years by SAS-2, COS-B and EGRET, unveiling very promising timing residuals (Mattox et al, 1997). The many "firsts" of Geminga are summarized in Table 1.

Quite surprisingly, some of the key parameters of Geminga are now known with an accuracy better than available for the Crab pulsar. This is due in part to the 
TABLE 1. GEMINGA(1973-present)

\begin{tabular}{|l|}
\hline - $1^{\text {st }}$ unidentified $\gamma$-ray source \\
\hline - $1^{\text {st }}$ INS discovered through high-energy emission and identified through its X and $\gamma$-rays \\
- $1^{\text {st }}$ INS identified without the help of radio astronomy \\
\hline - $1^{\text {st }}$ INS optically identified through its proper motion \\
- $1^{\text {st }}$ INS the distance of which is measured through its optical parallax \\
\hline - $1^{\text {st }}$ direct view in optical/UV of the surface/photosphere of a NS \\
- $1^{\text {st }}$ evidence for an atmosphere surrounding NS crust \\
- $1^{\text {st }}$ direct measurement of the surface magnetic field of an INS \\
\hline - $1^{\text {st }}$ INS the timing parameters of which are determined solely by high energy $\gamma$-ray data \\
- $1^{\text {st }}$ optical measurement of absolute position of an INS within 40 mas \\
(This leads to the first measurement of the braking index of a $10^{5}$ y old NS) \\
\hline - $1^{\text {st }}$ evidence (together with PSR0656+14 and PSR 1055-58) of an INS with a \\
two-component X-ray emission \\
\hline
\end{tabular}

20 year long chase (see Bignami and Caraveo, 1996 for a review), in part to the remarkable stability of this object which rendered possible to phase together such a long time span of $\gamma$-ray data.

\section{A STRATEGY FOR THE FUTURE}

There is no question that a sizeble fraction of the EGRET UGOs are galactic. Of these. It is reasonable to expect at least several to be radio-quiet INSs, since no further radio pulsars can be identified. Also, no other compact (or star-like) class of galactic objects has yet been identified with certainty as a $\gamma$-ray emitter. It make sense, therefore, to single out further $\gamma$-ray INSs even if the process might be difficult and tiresome, as in the case of Geminga. The potential reward will obviously be a better understanding of the radio-quiet, $\gamma$-ray loud INSs as a class. This would yield a precious addition to the general neutron star scanty phenomenology. Furthermore, a dedicated search process like that required to nail down an INS might well yield, as a bonus, new identifications of $\gamma$-ray objects of serendipitous nature.

To plan a strategy for such a search it is as easy as it is difficult to predict, with any confidence, its probability of success. This is to say that the only obvious way forward is one similar to the "Geminga chase" (Bignami and Caraveo, 1996). At the same time, we know from the start that, although possible Geminga-like, the majority of the UGOs must differ significantly from Geminga itself. The main difference will be in the absolute value of their $\gamma$-ray luminosity: objects as (relatively) faint as Geminga, will never be seen at the distances (several kpc) that UGOs must have to show their narrow latitude distribution. This could be due to differences in ages, which in term would yield different ratios of the power emitted in thermal versus non-thermal processes, both in optical and X-rays. This will impact on INS visibility. 
Nevertherless, until a better one is found, the UGO identification strategy can only be as follows:

1- map the UGO boxes with X-ray imaging devices

2- select those few sources which have a very high $F_{x} / F_{v}$

3- search for possible X-ray pulsations

4- go for optical IDs, using all possible methods, not forgetting proper motion.

With respect to the Geminga chase, carried out in the '80s and early '90s, steps 1 and 4 now benefit of a far deeper penetration in the sky owing to existing (and upcoming) orbiting and ground based telescopes. In the next couple of years, for example, the EPIC instrument on ESA's XMM will be operational as will be (at least) UT1 of ESO's VLT. Their joint usage, if well thought out, will improve by at least three to four magnitudes on the ROSAT/NTT combination, which did much of the work on Geminga. The extremely accurate relative astrometry, possible with the new instruments on HST will detect the much smaller proper motions (and parallaxes) of more distant INSs.

It should be exciting to see, within the next 5 years, how many more Geminga will be seen and if indeed a new different galactic population, other than INSs, is needed to explain EGRET's UGOs.

\section{REFERENCES}

Bertsch D.L., et al. 1992, Nature 357, 306

Bignami, G.F.,Caraveo,P.A., and Lamb, R.C. 1983, Ap.J., 272, L9

Bignami, G.F. and Hermsen W. Ann. Rev. Astr. Astrophys., 21,67

Bignami,G.F., Caraveo,P.A., Paul, J.A., Salotti,L. and Vigroux, L. 1987, Ap.J., 319, 358

Bignami, G.F., and Caraveo, P.A. 1992, Nature, 357, 287

Bignami, G.F., Caraveo, P.A., and Mereghetti, S. 1993, Nature, 361,704

Bignami G.F., Caraveo, P.A., Mignani R., Edelstein J. and Bowyer S. 1996, Ap.J., 456,L111

Bignami G.F., and Caraveo P.A. 1996, Ann. Rev. Astr. Astrophys., 34,331

Bignami G.F. 1997 Adv. Space Research, in press

Caraveo P.A., Bignami G.F., Mignani R., and Taff L.G. 1996, Ap.J., 461,L91

Caraveo P.A., Bignami G.F., Trümper J.A. 1996, A \& A Review, 7,209

Caraveo P.A.,et al. 1997, ESA SP 402, in press

ESA 1997, The Hipparcos and Tycho Catalogues ESA SP 1200

Goldoni P., Musso, C., Caraveo P.A., Bignami ,G.F. 1995 A \& A 298,535

Halpern, J.H., and Tytler, D. 1988, Ap.J., 330, 201

Halpern, J.P., and Holt, S.S. 1992, Nature, 357, 222

Kanbach, G. 1997 Adv. Space Research, in press

Fichtel, C.E. et al. 1975 Ap.J., 198,163

Mattox J.R., Halpern J.P. and Caraveo P.A. 1996, A \& A Suppl, 120C, 77

Mattox J.R., Halpern J.P. and Caraveo P.A. 1997 Ap.J. in press

Mattox J.R. et al 1992 Ap.J., 103,638 
Mukherjee R. et al 1995 Ap.J., 441, L61

Nel H.I. et al. 1996 Ap.J., 465,898

Nice D.J. and Sayer R.W. 1997 Ap.J., 476,261

Taylor,J., Manchester, R.M. Lyne A.G. 1993, Ap.J. Suppl., 88,529

Thompson D.J. et al, 1992 Nature 359,615

Thompson D.J. et al, 1995 Ap.J. Suppl. 101,259

Thompson D.J. et al, 1996 Ap.J. Suppl. 107,277 